\title{
Evaluation of a hybrid small caliber vascular graft in a rabbit model
}

Qin Fang, MD, ${ }^{\text {a }}$ Tianxiang Gu, MD, ${ }^{\mathrm{a}}$ Jun Fan, PhD, ${ }^{\mathrm{b}}$ Yuanming Zhang, MD, ${ }^{\mathrm{a}}$ Yongchao Wang, MD, Ye Zhao, MD, and Pan Zhao, $\mathrm{PhD}^{\mathrm{b}}$

\section{ABSTRACT}

Objective: A hybrid small-caliber artificial vascular graft based on bilayer porcine small intestinal submucosa (SIS) with curdlan and dipyridamole mixture film serving as the so-called sandwich filler was developed for biological performance evaluation. We evaluated the performance of the graft and filler.

Methods: SIS was coated with heparin by 1-ethyl-3-(3-dimethylaminopropyl) carbodiimide. Curdlan acted as the carrier of dipyridamole. Three types of graft tubes ( $2 \mathrm{~mm}$ internal diameter and $20 \mathrm{~mm}$ in length) were manufactured: bilayer SIS with $10 \%$ curdlan $+10 \%$ dipyridamole mixture film (SCD), bilayer SIS with $10 \%$ curdlan film (SC), and monolayer SIS (S). The remodeling characteristics of the grafts were evaluated by implanting them as bypass in rabbit carotid arteries for 2 and 3 months. Each group contained 16 rabbits, and 16 nonsurgical rabbits served as the control group.

Results: Eight rabbits of each group, including the graft occluded group, were killed at 2 months and the others were killed at 3 months. Follow-up showed that all 8 grafts in SCD group were patent at 2 months. Six of 16 grafts in the SC group and 5 of 16 grafts in the S group were occluded at 2 months. One of 8 SCD grafts were occluded at 3 months and the patent showed a confluent endothelium without intimal hyperplasia. The neointima layer was composed of circumferentially aligned vascular smooth muscle cells. At 3 months, SC and $\mathrm{S}$ group grafts showed incomplete endothelialization and varying degrees of mural thrombus, accompanied by occlusion in the SC group (3 of 8) and $\mathrm{S}$ group (2 of 8).

Conclusions: The novel hybrid small caliber artificial vascular graft exhibited an improvement in revascularization resulting in high patency rate. (J Thorac Cardiovasc Surg 2020;159:461-73)

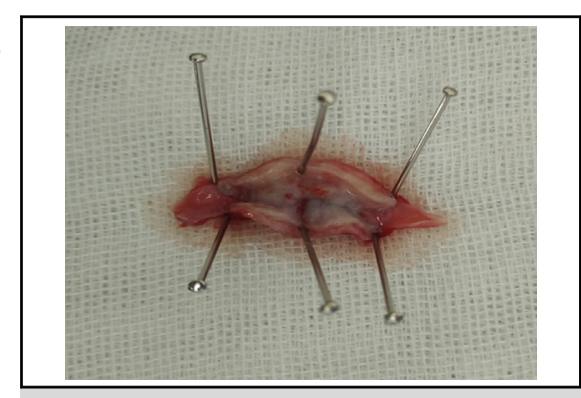

At 3 months, the lumen of a manufactured graft tube consisting of bilayer SIS with $10 \%$ curdlan $+10 \%$ dipyridamole mixture film filling was smooth, with no anastomotic stenosis.

\section{Central Message}

A hybrid graft based on bilayer heparinized SIS and filling composed of $10 \%$ curdlan $+10 \%$ DIP mixture film showed high patency rates in rabbit carotid artery bypass implantation models.

\section{Perspective}

Heparin coating and loading of DIP could improve the patency rate of in vivo implantation of a novel hybrid small caliber artificial vascular graft "sandwich."

See Commentaries on pages 474 and 476.
Widely used synthetic materials such as expanded polytetrafluoroethylene and nylon have been used in clinical settings as large-diameter artificial vascular grafts, but their applications in small arteries are limited because

\footnotetext{
From ${ }^{\mathrm{a} C a r d i a c}$ Surgery, First Hospital of China Medical University, Shenyang, China; and ${ }^{b}$ Department of Tissue Engineering, China Medical University, Shenyang, China.

Supported by the National Natural Science Foundation of China (contract No. 81770467), and the Department of Tissue Engineering, China Medical University, Shenyang, China.

Drs Fang and Fan contributed equally to this work.

Received for publication Oct 5, 2018; revisions received Feb 16, 2019; accepted for publication Feb 20, 2019; available ahead of print April 9, 2019.

Address for reprints: Tianxiang Gu, MD, Cardiac Surgery, First Hospital of China Medical University, 155 Nanjing N, Shenyang, China (E-mail: gtxcmu1@163. com).

$0022-5223 / \$ 36.00$

Copyright (c) 2019 by The American Association for Thoracic Surgery

https://doi.org/10.1016/j.jtcvs.2019.02.083
}

of poor hemocompatibility or biocompatibility. ${ }^{1}$ Small intestinal submucosa (SIS) is among the biomaterials that has been tried as a vascular substitute as far back as 1965 , but the biomaterial produced variable patency $(0 \%$ to $100 \%$ ) mainly due to thrombosis or failure due to dilation and dissection. ${ }^{2}$

Curdlan is a natural polysaccharide composed of $1,3-\beta$-linked D-glucose. A more elastic, stronger gel can be produced via heating of an aqueous solution to high

- Scanning this $\mathrm{QR}$ code will take you to the article title page to access supplementary information.

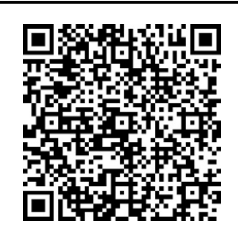




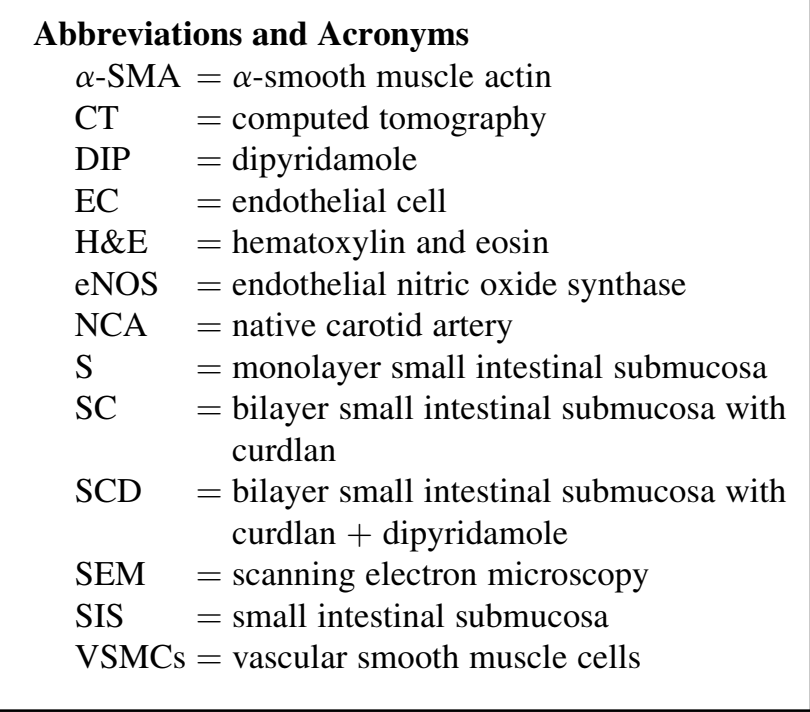

temperatures $\left(\mathrm{eg},>80^{\circ} \mathrm{C}\right)$ at adequate concentrations. ${ }^{3}$ It is nontoxic. ${ }^{4}$ The polysaccharide curdlan and its derivatives have a high latent capacity for pharmaceutical applications as a drug delivery system.

Dipyridamole (DIP) is an antiplatelet agent. Wakabayashi and colleagues ${ }^{6}$ showed that DIP had an inhibitory effect on the initiation of atherosclerosis elicited by leukocytes as well as that elicited by activated platelets. Furthermore, Punnakitikashem and colleagues ${ }^{7}$ showed that DIP had a positive effect on endothelial cell (EC) growth. Heparin sodium is widely used in clinical settings and it has been claimed often that heparinized materials possess microenvironment thromboresistance at the material-blood interface. ${ }^{8}$

Here we present a hybrid small caliber artificial vascular graft based on bilayer heparinized SIS, with curdlan and DIP mixture film as the filler sandwiched between the layers of SIS as the drug delivery system. Remodeling of the graft was assessed by rabbit carotid artery bypass implantation model.

\section{MATERIALS AND METHODS SIS Preparation}

SIS preparation procedure was according to Abraham's method. ${ }^{9}$ The effectiveness of decellularization was confirmed by hematoxylin and eosin (H\&E) staining. DNA was extracted according to the steps of DNA extraction kit (Tiangen Biotech, Beijing, China) and the DNA content was detected by multifunctional enzyme-linked immunoassay instrument (Tecan Infinite 200, Männedorf, Switzerland) to calculate the residual DNA to dry weight ratio.

\section{Immobilization of Heparin onto SIS}

For heparin saturated immobilization, a $15-\mathrm{cm}$ SIS patch was immersed in $50 \mathrm{~mL}$ 2-(N-morpholino) ethanesulfonic acid buffer (SunShine, Nanjing, China) with $\mathrm{pH} 1.5$, comprising $20 \mathrm{mM}$ N-hydroxysuccinimide (Best, Chendu, China), $20 \mathrm{mM}$ 1-ethyl-3-(3-dimethylaminopropyl) carbodiimide (Macklin, Shanghai, China), and heparin sodium
(12,500 U) (Qianhong Bio-pharma, Changzhou, China) and gently agitated for 36 hours at $37^{\circ} \mathrm{C} .^{10}$

\section{Graft Preparation}

A solution of $10 \%$ curdlan (Sigma Aldrich, St Louis, Mo) or $10 \%$ curdlan $+10 \%$ DIP (Sigma Aldrich) mixture film of thickness of $200 \mu \mathrm{m}$ was made in glass dishes and the hybrid suspensions were sterilized under high-pressure steam. A patch of suitable size heparinized SIS was rolled around a 2-mm diameter quartz rod. The SIS mucosal side was identified and served as the blood-contacting surface. The bilayer SIS with curdlan + DIP (SCD) or bilayer SIS with curdlan (SC) graft was made by wrapping 2 layers of SIS on the rod with a $10 \%$ overlap seam for suture. Size of $20 \times 8 \mathrm{~mm} 10 \%$ curdlan or $10 \%$ curdlan $+10 \%$ DIP mixture film served as the sandwich filler. Continuous round-trip sutures of 8-0 prolene line were applied to the seam with about $0.5 \mathrm{~mm}$ of interval (Figure 1). The suture technique was also applicable to monolayer SIS (Figure 1, Al). The distance of the midpoint of the graft opening was $2 \mathrm{~cm}$. The two ends of the graft retained a single layer structure with a distance $1 \mathrm{~mm}$ from the end to match rabbit's native carotid artery (NCA) and $45^{\circ}$ oblique angle convenient for bypass anastomosis.

\section{Sample Size Selection, Animal Grouping, and Surgery}

The sample size was determined by patency rate. Our preliminary experimental results demonstrated that the difference in 3-month patency rate between SCD and monolayer SIS (S) or SC was about $50 \%$ ( 9 of 10 $\mathrm{SCD}, 4$ of $10 \mathrm{~S}$, and 4 of $10 \mathrm{SC}$ were patent at 3 months). To detect a difference of $50 \%$ between the treatment groups with a power of 0.8 , beta of 0.2 , and an alpha of 0.05 , a sample size of 13 per treatment group was required. Finally, 16 animals were used in each group for more accuracy. The experiment was approved by the Animal Experiments Ethical Committee of China Medical University and carried out in accordance with National Institutes of Health guide for the care and use of Laboratory animals. Sixty-four male New Zealand rabbits (body weight, 2.5-3 kg, obtained from China Medical University laboratory animal center) were assigned randomly into SCD, SC, S, or control group with 16 cases in each group. Surgical rabbits were anesthetized by the administration of $3 \%$ pentobarbital sodium (Sigma Aldrich) through intraperitoneal injection according to $45 \mathrm{mg} / \mathrm{kg}$. After median cervical incision, the grafts were connected to left carotid arteries by end-to-side anastomosis with $8-0$ prolene suture line. The native carotids were ligated between the proximal and distal anastomosises to prevent competitive flow (Figure 1, B1 through $B 3$ and Video 1). One died of respiratory depression on the day after $\mathrm{S}$ graft implantation. One died of anorexia 3 days after SC graft implantation, and no death occurred in the SCD and control groups. Deaths were excluded from the 64 cases.

\section{Doppler Ultrasound and Computed Tomography Angiography}

The inner diameters (middle point), patency and appearance of the bypass grafts were evaluated by color Doppler (Philips Ie33, Amsterdam, Netherlands) or computed tomography (CT) scanning (64 row dual source CT; Siemens, Berlin, Germany).

\section{Explantation and Measurement of Grafts}

Grafts occluded at 2 months in each group were explanted. The remaining of each group were randomly collected added up to 8 cases and explanted at 2 months. Other 8 cases of each group were fed for 3 months and explanted. The patent and incompletely occluded grafts were cut into 3 equal length parts (proximal, middle, and distal fragments). Moreover, the thickness of the graft wall was measured on 6 different parts $(2,4,6,8,10$, and 12 o'clock positions) at the proximal end of the middle fragment. The middle fragments were used for DIP-releasing analysis and 

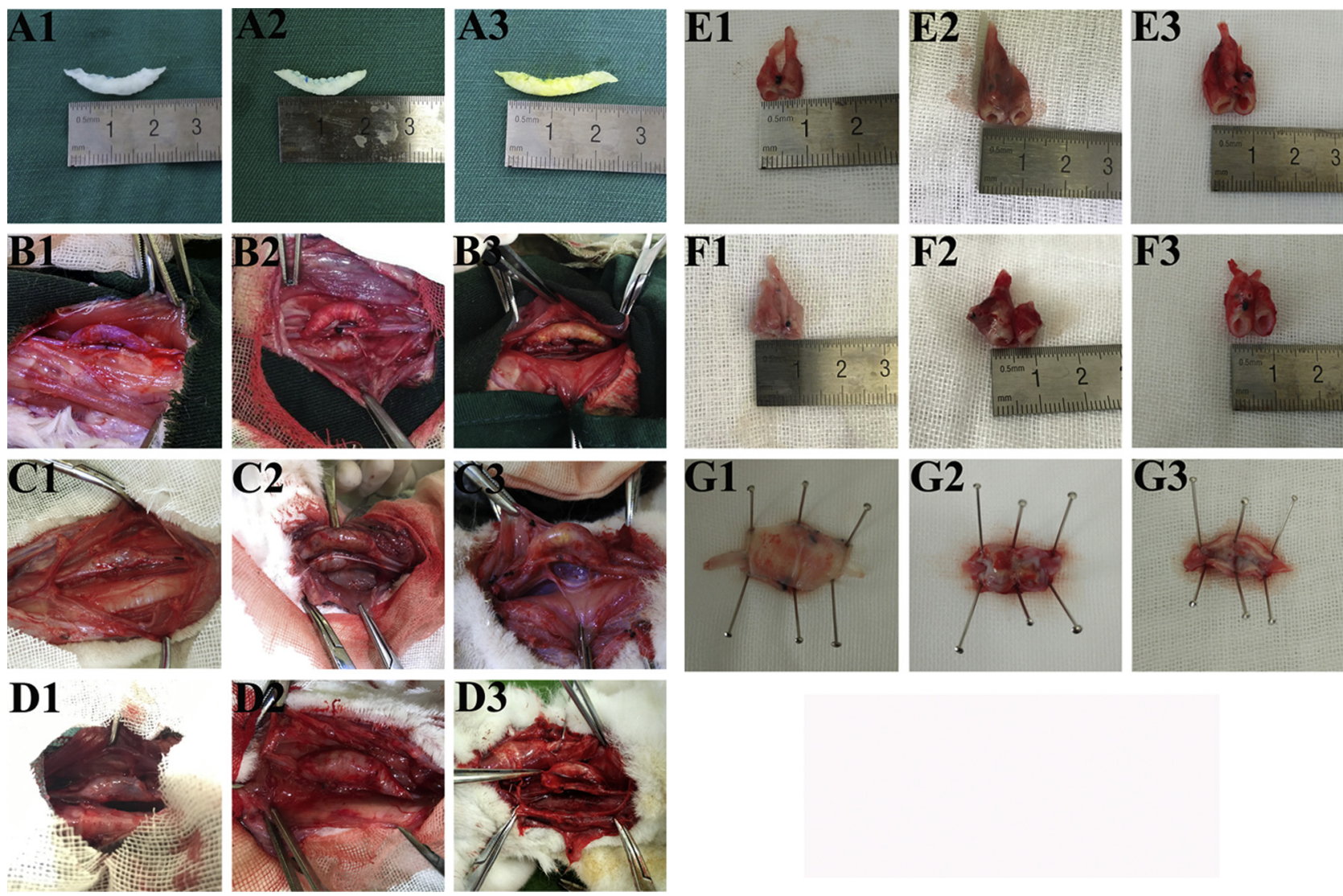

FIGURE 1. Graft preparation, implantation, explantation, and evaluation. A1, Graft preparation of monolayer small intestinal submucosa (SIS) (S). A2, Graft preparation of bilayer SIS with 10\% curdlan film (SC). A3, Graft preparation of bilayer SIS with 10\% curdlan + 10\% dipyridamole (DIP) mixture film (SCD). The distance of the midpoint of the graft opening was $2 \mathrm{~cm}$. B1, Graft implantation of S. B2, Graft implantation of SC. B3, Graft implantation of SCD. End-to-side anastomosis was used and native carotid artery was ligated between the proximal and distal anastomosis. C1, Graft explantation of S at 2 months. C2, Graft explantation of SC at 2 months. C3, Graft explantation of SCD at 2 months. D1, Graft explantation of S at 3 months. D2, Graft explantation of SC at 3 months. D3, Graft explantation of SCD at 3 months. Incompletely absorbed DIP can be seen through adventitia of SCD at 2 months. E1, A representative transectional picture of explants of S at 2 months. E2, A representative transectional picture of explants of SC at 2 months. E3, A representative transectional picture of explants of SCD at 2 months. F1, A representative transectional picture of explants of S at 3 months. F2, A representative transectional picture of explant of SC at 3 months. F3, A representative transectional picture of explant of SCD at 3 months. S at 3 months has larger inner diameter and thinner wall than S at 2 months. SC at 3 months has smaller inner diameter and thicker wall than SC at 2 months. SCD at 3 months has similar size inner diameter but thinner wall compared with SCD at 2 months. Incompletely absorbed DIP can be seen from the walls of SCD at 2 months and SCD at 3 months. G1, Representative longitudinal picture of explant of S at 3 months. G2, Representative longitudinal picture of explant of SC at 3 months. G3, Representative longitudinal picture of explant of SCD at 3 months (the lumen of SCD at 3 months was smooth, with no anastomosis stenosis). The lumen of $\mathrm{S}$ at 3 months was dilated. The lumen of SC at 3 months was rough.

protein extract for Western blot procedures. The proximal and distal fragments were then placed in $4 \%$ paraformaldehyde for staining or $2.5 \%$ glutaraldehyde for scanning electron microscopy (SEM), respectively. The animals were killed by overdose injection of pentobarbital sodium through ear vein after explanation of the grafts.

\section{In vivo DIP Release}

Seven explanted SCD-3 month middle segments were crosscut into 2 halves, half of proximal segments were homogenized by using a superfine homogenizer, respectively. The $\mathrm{v} / \mathrm{v}$ ratio of each tissue to phosphate-buffered saline was 1:3. Followed by centrifugation, equal volume of $100 \%$ ethanol was added to the supernatant. DIP content was quantified by detecting fluorescence intensity. The fluorescence intensity was then recorded at $295 \mathrm{~nm}$ (excitation) and $485 \mathrm{~nm}$ (emission) by multifunctional enzyme-linked immunoassay instrument. A standard curve was performed using a series of known DIP concentrations in $50 \%$ alcohol/phosphate-buffered saline solution. ${ }^{11}$

\section{Histology}

Graft fragments fixed and embedded in paraffin, were sectioned at $6 \mu \mathrm{m}$ thickness and processed for H\&E, Masson's trichrome, orcein staining, and immunofluorescence evaluation. ${ }^{12}$ Collagen was quantified by Masson's trichrome staining. Images of graft from 3 to 5 consecutive cross-sections for each animal were collected at equal exposure conditions using an Olympus BX51 microscope (Olympus, Tokyo, Japan). Green-stained collagen areas were quantified with Image $\mathbf{J}$ (National Institutes of Health, Bethesda, Md) from 4 to 5 regions per section. 


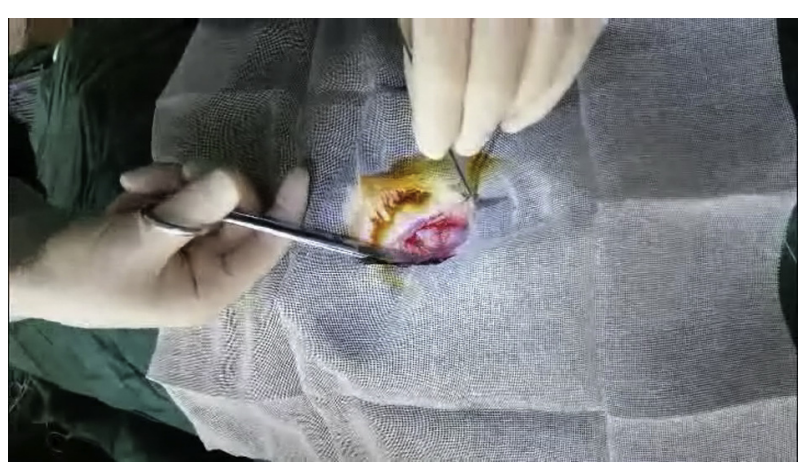

VIDEO 1. After median cervical incision, the left native carotid artery (NCA) was separated from surrounding tissues and heparin was injected into left common jugular vein. The NCA was clamped at the proximal and distal end. The longitudinal incision length of the anterior wall of the NCA was $3 \mathrm{~mm}$ and the distance between the 2 incisions was $2 \mathrm{~cm}$. Continuous suture with 8-0 suture line connects the both ends of the graft to NCA by end-to-side anastomosis. Video avaialbe at: https://www.jtcvs. org/article/S0022-5223(19)30550-1/fulltext.

\section{Immunofluorescence}

Primary antibodies mouse anti-rabbit CD31 (1:200) (Abcam, Cambridge, Mass) and mouse anti-rabbit $\alpha$-SMA (1:200) (Novus, Littleton, Colo) were used. The nuclei were counterstained with DAPI (Beyotime, Shanghai, China). Images of graft from 3 to 5 consecutive cross-sections for each animal were acquired under a fluorescence microscope (Olympus DP80).

To identify the degree of endothelialization of the graft lumen surface, the proportion of CD31-positive parts to the total lumen surface circumference (the endothelialization rate) was analyzed using Image $\mathbf{J} .{ }^{13}$

\section{Western Blot Analysis}

Briefly, the removed graft segments were lysed in radioimmunoprecipitation assay buffer (Beyotime). The protein concentration was measured by bicinchoninic acid protein assay kit (Beyotime). Proteins of equal quality were added in $8 \%$ to $12 \%$ sodium dodecyl sulfate-polyacrylamide gel (CW Biotech, Beijing, China) to start electrophoresis. The polyvinylidene fluoride membrane was immunoblotted by using endothelial nitric oxide synthase (eNOS), $\alpha$-smooth muscle actin ( $\alpha$-SMA) (Novus), and $\beta$-tubulin (Bioss, Beijing, China) as an internal control.

\section{Statistical Analysis}

Graft patency rate was designed as the primary outcome. The statistical analyses were performed using the SPSS version 13.0 software package (IBM-SPSS Inc, Armonk, NY). Data are reported as mean \pm standard deviation when normally distributed (residual DNA of SIS and DIP release) or median (interquartile range) when nonnormally distributed (graft wall thickness, inner diameter, collagen volume fraction, endothelialization rate, and the expression of eNOS and $\alpha$-SMA) and percentages for categorical data otherwise. Nonparametric Mann-Whitney $U$ test was used for nonnormally distributed quantitative variables between different groups as well as different times. Fisher exact test was used to analyze patency rate comparison.

\section{RESULTS}

\section{SIS Preparation}

H\&E staining showed the mucosal side of SIS before decellularization was smooth, the muscular side was rough, and residual blue nuclei were still visible. But no signs of remaining nuclear material could be seen after decellularization (Figure 2, $A$ and $B$ ). SEM observation showed that the structure of the muscular side became loose 3-dimensional reticulated stereostructures (Figure 2, $C$ and $D$ ). The residual DNA of SIS after decellularization was $39.3 \pm 10.5 \mathrm{ng} / \mathrm{mg}$, which was $<1 \%$ of fresh SIS DNA $(6473.2 \pm 537.3 \mathrm{ng} / \mathrm{mg})$.

\section{Curdlan and DIP Mixture Film}

Vacuum freeze-dried curdlan and DIP mixture film was observed under SEM, which showed 3-dimensional spatial structure with pore size of 20 to $250 \mu \mathrm{m}$. DIP particles were homogeneously adhered to porous space structure of gel (Figure 2, $E$ and $F$ ).

\section{DIP Release Analysis}

The results showed that approximately about $53.39 \% \pm 3.52 \%$ of DIP was released at 2 months; $91.01 \% \pm 4.79 \%$ was released at 3 months.

\section{Evaluation of Graft Patency by Doppler Ultrasound, CT Angiography, and Gross Analysis}

Doppler showed that all SCD-2 months were patent, and 7 of 8 SCD-3 months were patent (Figure 3, B4). However, 6 of $16 \mathrm{SC}$ grafts and 5 of $16 \mathrm{~S}$ grafts occluded at 2 months, among which 2 of SC-2 months and 1 of S-2 months occluded incompletely through cross-sectional observation. The explanted grafts of each group were shown in (Figure 1, E1 through E3) and (Figure 1, F1 through F3). The lumens of explanted patent SCD at 3 months were smooth. The lumens of explanted patent SC at 3 months and $\mathrm{S}$ at 3 months showed varying degrees of mural thrombus, accompanied by SC at 3 months occlusion ( 3 of 8 ) and $S$ at 3 months occlusion (2 of 8). These signs were consistent with Doppler results (Figure 3, B2 through $B 4)$. Incompletely absorbed DIP could be seen from the walls of SCD-2 month and SCD-3 month explants. Representative longitudinal pictures of 3 month-explanted grafts of each group are shown in Figure 1, Gl through G3. The lumen of patent SCD-3 months was smooth with no anastomosis stenosis. The lumen of S-3 months was dilated obviously compared with NCA. The lumen of SC3 months was rough and uneven. Compared with S-3 months and SC-3 months, intima of SCD-3 months had more compact and regular distribution of ECs, which was similar to NCA (Figure 3, Al through A4).

The graft wall thickness is shown in Figure $4, A$ and $B$, and Table 1 . All grafts at 2 months were significantly thicker than NCA at 2 months $(P<.05)$. SCD-3 months (median, $651 \mu \mathrm{m}$; IQR, 560-683 $\mu \mathrm{m}$ ) was thinner than SCD-2 months (median, $1076 \mu \mathrm{m}$; IQR, 943-1216 $\mu \mathrm{m})(P=.001)$. SC-3 months (median, $1276 \mu \mathrm{m}$; IQR, 1264-1287 $\mu \mathrm{m}$ ) was thicker than SC-2 months (median, $979 \mu \mathrm{m}$; IQR, 940-1037 $\mu \mathrm{m})(P=.016)$. 

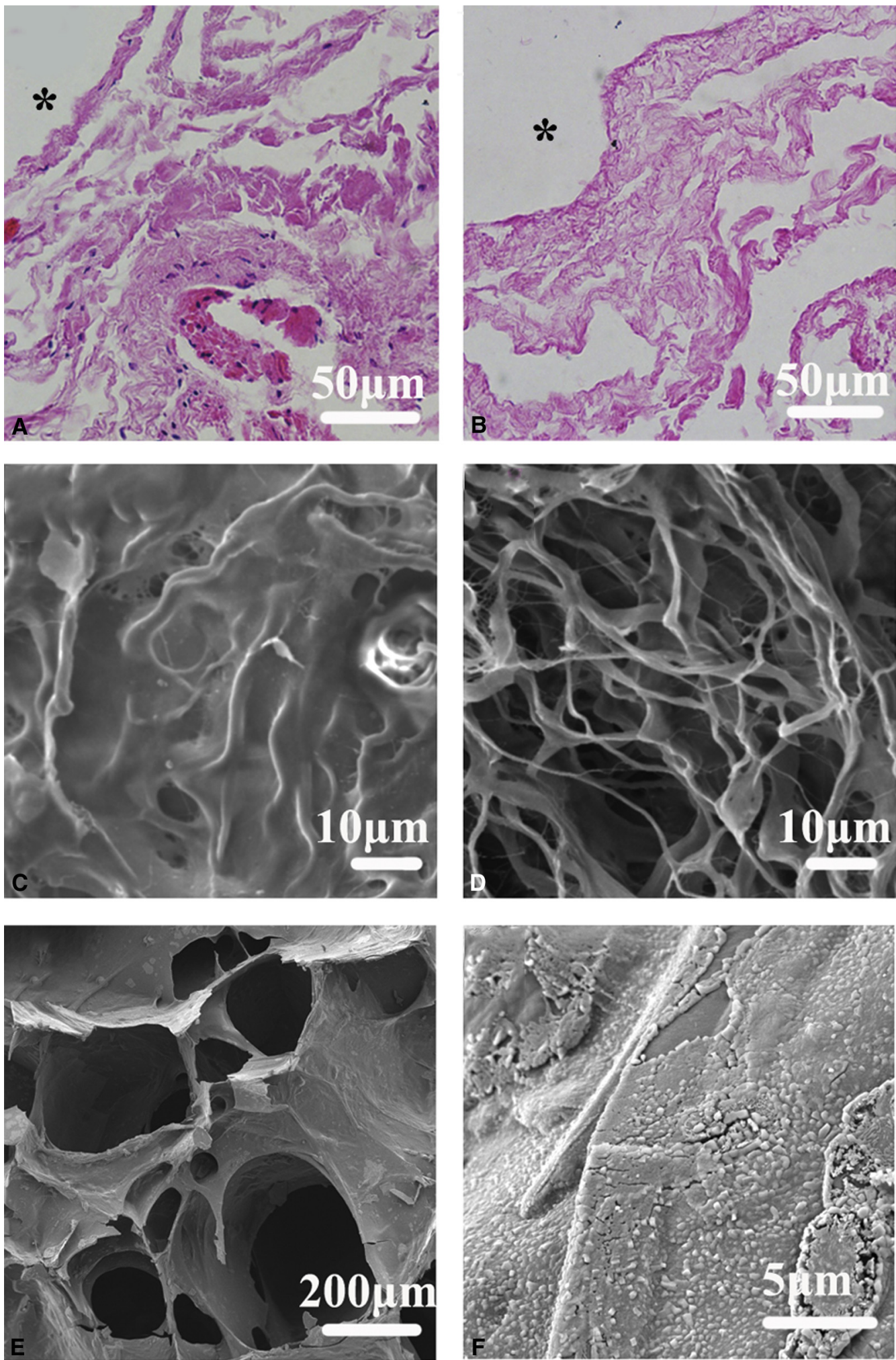

FIGURE 2. Structure characterization of small intestinal submucosa (SIS) and film. A, Hematoxylin and eosin staining of fresh SIS prior decellularization showed smooth mucosal side, rough muscular side, and residual blue nuclei. B, After decellularization, SIS showed no signs of remaining nuclear material. C, Muscular side of SIS before decellularization was rough, dense, and nonporous. D, After decellularization, muscular side of SIS became loose 3-dimensional reticulated stereostructure under scanning electron microscopy (SEM) (Bar: $10 \mu \mathrm{m})$. E, Vacuum freeze-dried curdlan and dipyridamole (DIP) mixture film was observed under SEM (Bar: $200 \mu \mathrm{m}$ ) and showed 3-dimensional spatial structure with pore size of 20 to $250 \mu \mathrm{m}$. F, Vacuum freeze-dried curdlan and DIP mixture film was observed under SEM (Bar: $5 \mu \mathrm{m})$ and showed DIP particles that were homogeneously adhered to the porous space structure of gel. *Mucosal side of SIS. 

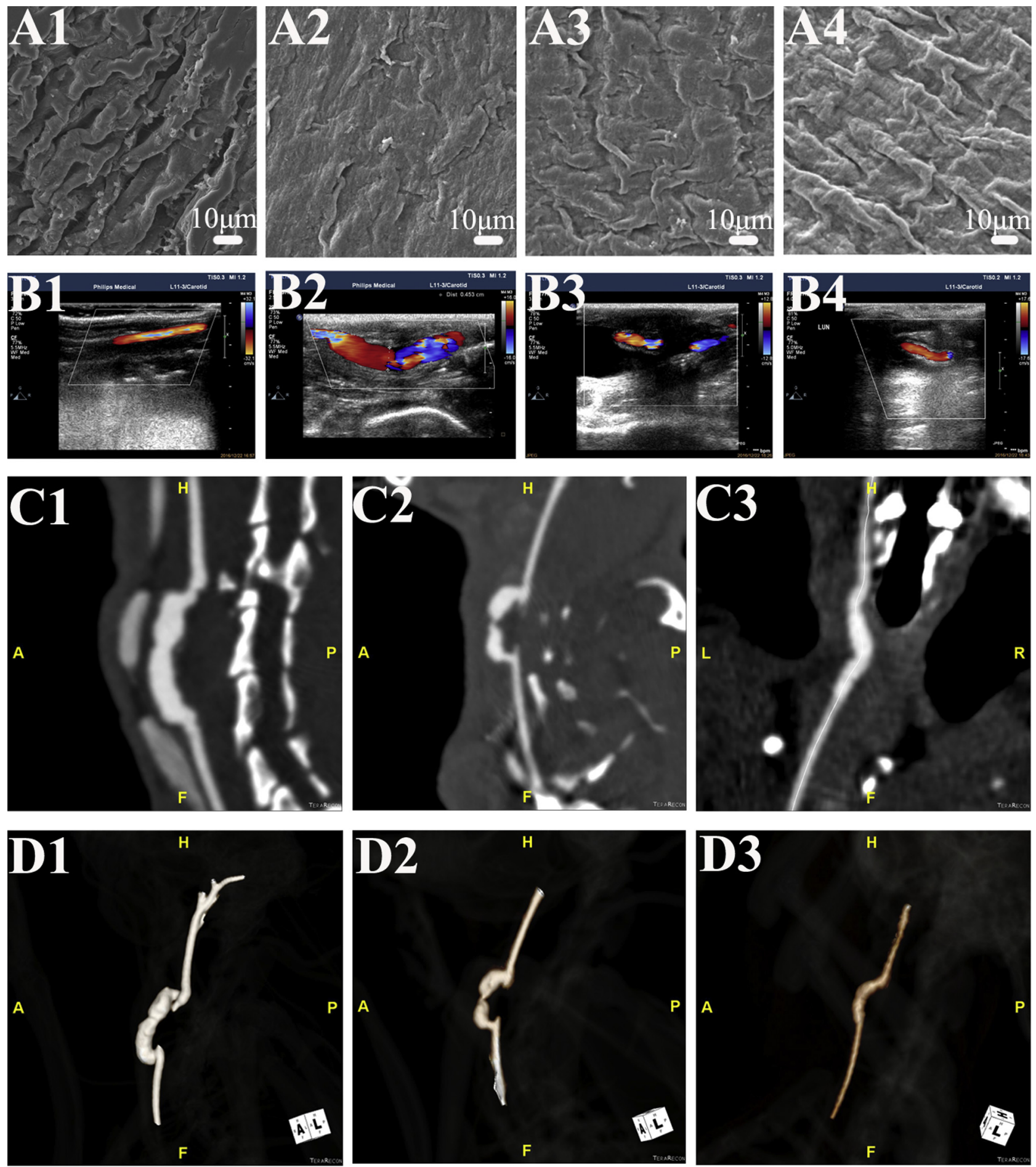

H

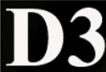

H
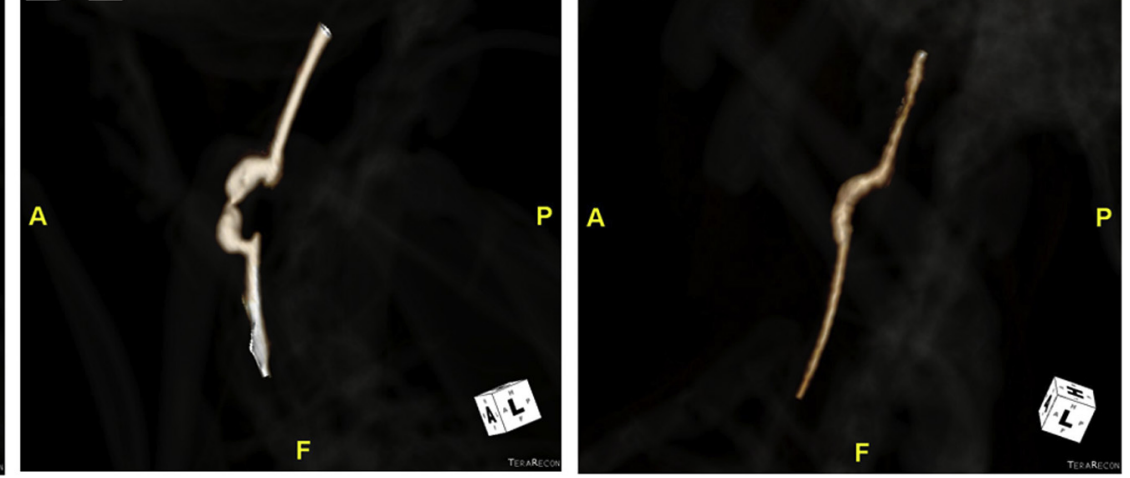

FIGURE 3. Evaluation of graft by scanning electron microscopy (SEM), Doppler ultrasound, and computed tomography (CT) angiography. A1, Intimal surface SEM images of native carotid artery (NCA). A2, Intimal surface SEM images of monolayer small intestinal submucosa (S) at 3 months. A3, Intimal surface SEM images of bilayer small intestinal submucosa with curdlan (SC) at 3 months. A4, Intimal surface SEM images of bilayer small intestinal submucosa with curdlan + dipyridamole (SCD) at 3 months. Compared with S at 3 months and SC at 3 months, SCD at 3 months had a more compact and regular distribution of endothelial cells, which was similar to NCA. B1, Doppler images of NCA. B2, Doppler images of S at 3 months. B3, Doppler images of SC at 3 months. B4, Doppler images of SCD at 3 months. The lumen of S at 3 months dilated obviously compared with NCA, SC at 3 months was uneven, and SCD at 3 months was smooth. C1 and D1, Computed tomography (CT) angiography images of S at 3 months. C2 and D2, CT angiography images of SC at 3 months. C3 and D3, CT angiography images of SCD at 3 months. The lumen of S at 3 months dilated inhomogeneously, SC at 3 months narrowed partially, and SCD at 3 months was patent and uniform. 

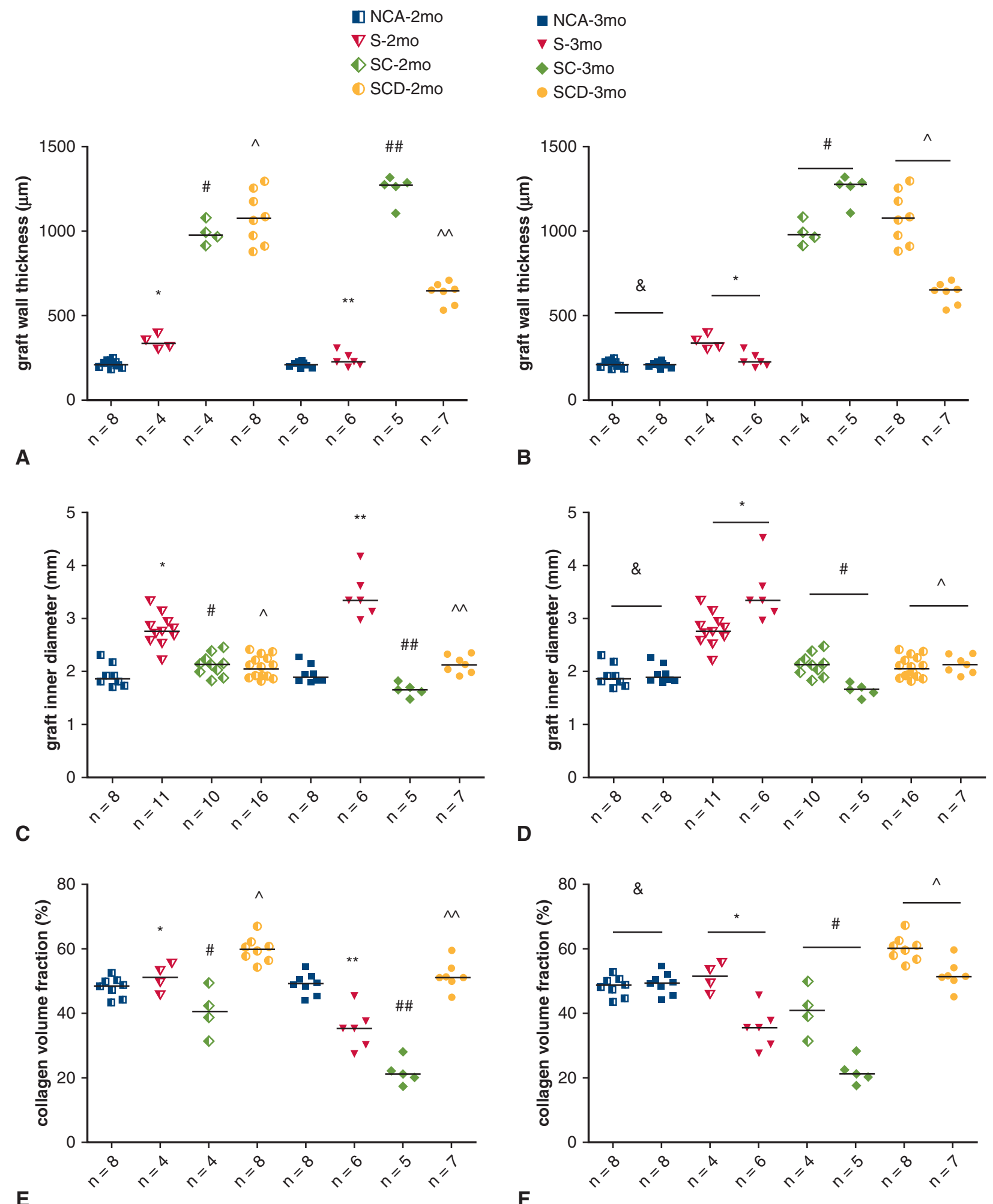

FIGURE 4. Changes of wall thickness, inner diameter, and collagen volume fraction in the transplanted graft. Nonparametric test Mann-Whitney $U$ test was used. A, Versus NCA at 2 months: $* P=.004, \# P=.004$, and ${ }^{\wedge} P=.001$. Versus NCA at 3 months: $* * P=.181, \# \# P=.002$, and ${ }^{\wedge} P=.001$. $\mathrm{B}, \& P=.878,{ }^{*} P=.019, \# P=.016$, and ${ }^{\wedge} P=.001 . \mathrm{C}$, Versus NCA at 2 months: $* P=.001, \# P=.068$, and ${ }^{\wedge} P=.052$. Versus NCA at 3 months: $* * P=.001, \# \#=.003$, and ${ }^{\wedge} P P=.054 . \mathrm{D}, \& P=.574, * P=.002, \# P=.001$, and ${ }^{\wedge} P=.413$. E, Versus NCA at 2 months: $* P=.283, \# P=.073$, and ${ }^{\wedge} P=.001$. Versus NCA at 3 months: ${ }^{*} * P=.003$, \#\#P=.002, and ${ }^{\wedge} \wedge P=.232 . \mathrm{F}, \& P=.574,{ }^{*} P=.01, \# P=.016$, and ${ }^{\wedge} P=.004 . N C A$, Native carotid artery; $S$, monolayer small intestinal submucosa; $S C$, bilayer small intestinal submucosa with curdlan; $S C D$, bilayer small intestinal submucosa with curdlan + dipyridamole. 
TABLE 1. Native carotid artery (NCA) graft wall thickness, inner diameter, and collagen volume fraction

\begin{tabular}{|c|c|c|c|}
\hline Variable & Graft wall thickness $(\mu \mathrm{m})$ & Graft inner diameter (mm) & Collagen volume fraction $(\%)$ \\
\hline $\mathrm{NCA}$ at $2 \mathrm{mo}$ & $212(197-228)(n=8)$ & $1.87(1.78-2.05)(\mathrm{n}=8)$ & $48.44(45.79-49.94)(n=8)$ \\
\hline $\mathrm{NCA}$ at $3 \mathrm{mo}$ & $212(197-224)(n=8)$ & $1.89(1.83-2.06)(\mathrm{n}=8)$ & $49.24(46.75-50.99)(\mathrm{n}=8)$ \\
\hline $\mathrm{S}$ at $2 \mathrm{mo}$ & $339(311-378)(\mathrm{n}=4)$ & $2.76(2.59-2.93)(\mathrm{n}=11)$ & $51.27(47.54-54.31)(\mathrm{n}=4)$ \\
\hline $\mathrm{S}$ at $3 \mathrm{mo}$ & $228(209-262)(n=6)$ & $3.35(3.13-3.61)(\mathrm{n}=6)$ & $35.37(30.25-37.61)(n=6)$ \\
\hline $\mathrm{SC}$ at $2 \mathrm{mo}$ & $979(940-1037)(n=4)$ & $2.14(1.99-2.23)(\mathrm{n}=10)$ & $40.62(35.06-45.98)(n=4)$ \\
\hline $\mathrm{SC}$ at $3 \mathrm{mo}$ & $1276(1264-1287)(\mathrm{n}=5)$ & $1.66(1.61-1.71)(\mathrm{n}=5)$ & $21.23(20.09-22.33)(\mathrm{n}=5)$ \\
\hline $\mathrm{SCD}$ at $2 \mathrm{mo}$ & $1076(943-1216)(\mathrm{n}=8)$ & $2.06(1.92-2.23)(\mathrm{n}=16)$ & $59.97(57.11-61.49)(\mathrm{n}=8)$ \\
\hline $\mathrm{SCD}$ at $3 \mathrm{mo}$ & $651(560-683)(\mathrm{n}=7)$ & $2.13(1.91-2.33)(\mathrm{n}=7)$ & $51.17(49.92-53.92)(\mathrm{n}=7)$ \\
\hline
\end{tabular}

Values are presented as median (interquartile range). $N C A$, Native carotid artery; $S$, monolayer small intestinal submucosa; $S C$, bilayer small intestinal submucosa with curdlan; $S C D$, bilayer small intestinal submucosa with curdlan + dipyridamole

The inner diameter is shown in Figure 4, $C$ and $D$, and Table 1. S-3 months (median, $3.35 \mathrm{~mm}$; IQR, 3.13-3.61 mm) was significantly larger than NCA-3 months (median, $1.89 \mathrm{~mm}$; IQR, $1.83-2.06 \mathrm{~mm})(P=.001)$. SC-3 months (median, $1.66 \mathrm{~mm}$; IQR, 1.61-1.71 mm) was significantly smaller than NCA-3 months (median, $1.89 \mathrm{~mm}$; IQR, 1.83-2.06 mm) $(P=.003)$.

The patency rates were showed in Table 2. Patency rates of S-2 months +3 months (9 out of 16 samples) and SC-2 months +3 months (7 out of 16 samples) were significantly lower than SCD-2 months +3 months (15 out of 16 samples) $(P=.019$ and .003 , respectively).

CT angiography images of S-3 months, SC-3 months, and SCD-3 months are shown in Figure 3. The lumen of S-3 months samples were dilated inhomogeneously, SC-3 months samples were narrowed partially, and SCD-3 months samples were patent and uniform.

\section{Collagen and Elastin Analysis}

Collagen was quantified by Masson's trichrome staining. Collagen positive area of grafts at 3 months were all less than grafts at 2 months (Figure 5, A2 through G2). For comparison of collagen volume fraction, SCD-2 months (median, 59.97\%; IQR, 57.11\%-61.49\%) was significantly higher than NCA-2 months (median, 48.44\%; IQR, 45.79\%-49.94\%) $(P=.001)$. S-3 months (median, $35.37 \%$; IQR, 30.25\%-37.61\%) and SC-3 months (median, 21.23\%; IQR, 20.09\%-22.33\%) were significantly lower than NCA-3 months (median, 49.24\%;

TABLE 2. Patency comparison between grafts

\begin{tabular}{lllc}
\hline & \multicolumn{3}{c}{ Patency(n) } \\
\cline { 2 - 4 } Graft & $\mathbf{2 ~ m o}$ & $\mathbf{3 ~ m o}$ & $\mathbf{2 ~ m o}+\mathbf{3}$ mo \\
\hline S & $11(16)$ & $6(8)$ & $9(16)^{*}$ \\
SC & $10(16)$ & $5(8)$ & $7(16) \dagger$ \\
SCD & $16(16)$ & $7(8)$ & $15(16)$ \\
\hline
\end{tabular}

Values are presented as $\mathrm{n}(\%)$. Fisher exact test was used. $S$, Monolayer small intestinal submucosa; $S C$, bilayer small intestinal submucosa with curdlan; $S C D$, bilayer small intestinal submucosa with curdlan + dipyridamole $* P=.019$ versus $\mathrm{SCD}-2 \mathrm{mo}+3 \mathrm{mo} . \dagger P=.003$ versus SCD-2 mo +3 mo.
IQR, $46.75 \%-50.99 \%)(P=.003$ and .002 , respectively $)$ (Figure 4, $E$ and $F$, and Table 1).

Elastin was evaluated by Orcein staining (Figure 5, A3 through G3). Typical fibrillar elastin can be seen in NCA, but no fibrillar elastin was found in the grafts.

\section{Tissue Remodeling and Cellularization}

Cross-sectional H\&E staining of the NCA and grafts are showed in (Figure 5, Al through G1). S-2 months had mural thrombus. S-3 months showed dilation. SC-2 months and SCD-2 months showed thick wall. SC-3 months had mural thrombus and thicker wall than SC-2 months. SCD-3 months had smooth lumen and thinner wall than SCD-2 months.

Presence of ECs on the surface of the lumens was verified by positive $\mathrm{CD} 31$ immunofluorescence staining (Figure 6, Al through $G 1$ ), which indicated that ECs were scattered in the lumens of S-2 months, S-3 months, SC-2 months and SC-3 months. ECs were almost continuously distributed in the lumens of NCA, SCD-2 months, and SCD-3 months.

The presence of vascular smooth muscle cells (VSMCs) was also confirmed by $\alpha$-SMA-specific antibody staining by immunofluorescence assay (Figure 6, $A 2$ through $G 2$ ), which indicated that VSMC layer of grafts at 3 months were thicker than grafts at 2 months. VSMC layer of SCD-2 months was thinner than S-2 months and SC-2 months, and SCD-3 months was thinner than S-3 months and SC-3 months. VSMC layer of SCD-3 months was more clearly organized, there was no intimal hyperplasia, and the new intima was composed of circumferentially aligned VSMCs.

For comparison of endothelialization rate, S-2 months (median, 36.3\%; IQR, 32.9\%-38.8\%) and SC-2 months (median, 35.9\%; IQR, 30.2\%-42.7\%) were significantly lower than SCD-2 months (median, 79.9\%; IQR, $73.5 \%-80.9 \%) \quad(P=.004$ and .004 , respectively $)$, S-3 months (median, 54.3\%; IQR, 50.4\%-57.1\%) and SC-3 months (median, 55.4\%; IQR, 53.7\%-57.5\%) were 

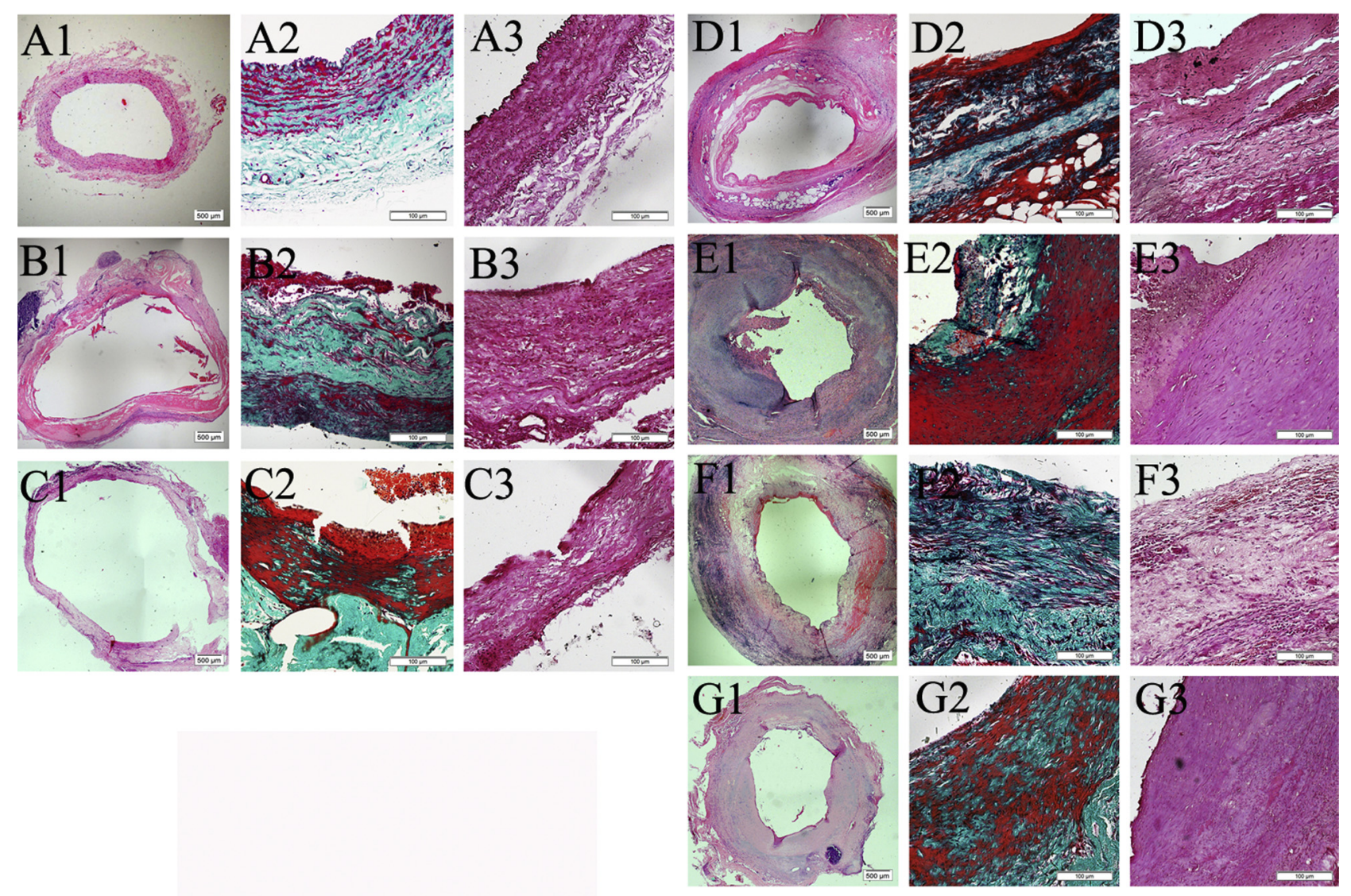

FIGURE 5. Histologic alterations in the grafts. A1, Hematoxylin and eosin (H\&E) staining of native carotid artery (NCA). B1, H\&E staining of monolayer small intestinal submucosa (S) at 2 months. C1, H\&E staining of S at 3 months. D1, H\&E staining of bilayer small intestinal submucosa with curdlan (SC) at 2 months. E1, H\&E staining of SC at 3 months. F1, H\&E staining of SCD at 2 months. G1, H\&E staining of bilayer small intestinal submucosa with curdlan + dipyridamole (SCD) at 3 months. S at 2 months showed mural thrombus, $\mathrm{S}$ at 3 months showed luminal dilation. SC at 3 months showed mural thrombus and thicker wall than SC at 2 months. SCD at 3 months showed smooth lumen and thinner wall than SCD at 2 months. A2, Masson's trichrome staining of NCA. B2, Masson's trichrome staining of S at 2 months. C2, Masson's trichrome staining of S at 3 months. D2, Masson's trichrome staining of SC at 2 months. E2, Masson's trichrome staining of SC at 3 months. F2, Masson's trichrome staining of SCD at 2 months. G2, Masson's trichrome staining of SCD at 3 months. Collagen positive areas of grafts at 3 months were all less than grafts at 2 months, but SCD at 3 months was similar to NCA. A3, Orcein staining of NCA. B3, Orcein staining of S at 2 months. C3, Orcein staining of S at 3 months. D3, Orcein staining of SC at 2 months. E3, Orcein staining of SC at 3 months. F3, Orcein staining of SCD at 2 months. G3, Orcein staining of SCD at 3 months. Typical fibrillar elastin can be seen in NCA, but no fibrillar elastin was found in any of the grafts.

significantly lower than SCD-3 months (median, 90.3\%; IQR, 84.4\%-91.9\%) ( $P=.001$ and .003, respectively). Grafts at 3 months were all higher than grafts at 2 months $(P<.05)$ (Table 3).

\section{Western Blot Analysis}

To assess the function of adherent ECs and behavior of SMCs in the graft, Western blots for eNOS and $\alpha$-SMA were performed. $\beta$-tubulin was used as an internal control (Figure 7, A). Quantitative analysis showed that expressions of eNOS and $\alpha$-SMA increased over time in the experimental groups.

The relative expression of eNOS was expressed by the ratio of eNOS gray value to internal control $\beta$-tubulin. The values are shown as median (range). eNOS/ $\beta$-tubulin of S-2 months (median, 0.071; IQR, 0.067-0.075) and
SC-2 months (median, 0.018; IQR, 0.015-0.021) were lower than SCD-2 months (median, 0.238; IQR, 0.225$0.252)(P=.004$ and .004 , respectively). eNOS/ $\beta$-tubulin of S-3 months (median, 0.088; IQR, 0.071-0.122) and SC3 months (median, 0.112; IQR, 0.102-0.123) were lower than SCD-3 months (median, 0.407; IQR, 0.387-0.471) $(P=.001$ and .003 , respectively) (Figure 7, $B$ and $C$, and Table 4).

$\alpha$-SMA $/ \beta$-tubulin of S-2 months (median, 1.776; IQR, 1.679-1.854) and SC-2 months (median, 1.627; IQR, 1.581-1.734) were higher than SCD-2 months (median, 0.803; IQR, 0.745-0.854) ( $P=.004$ and .004, respectively). $\alpha$-SMA $/ \beta$-tubulin of S-3 months (median, 2.373; IQR, 2.145-2.461) and SC-3 months (2.261 [2.227-2.294]) were higher than SCD-3 months (median, 1.434; 

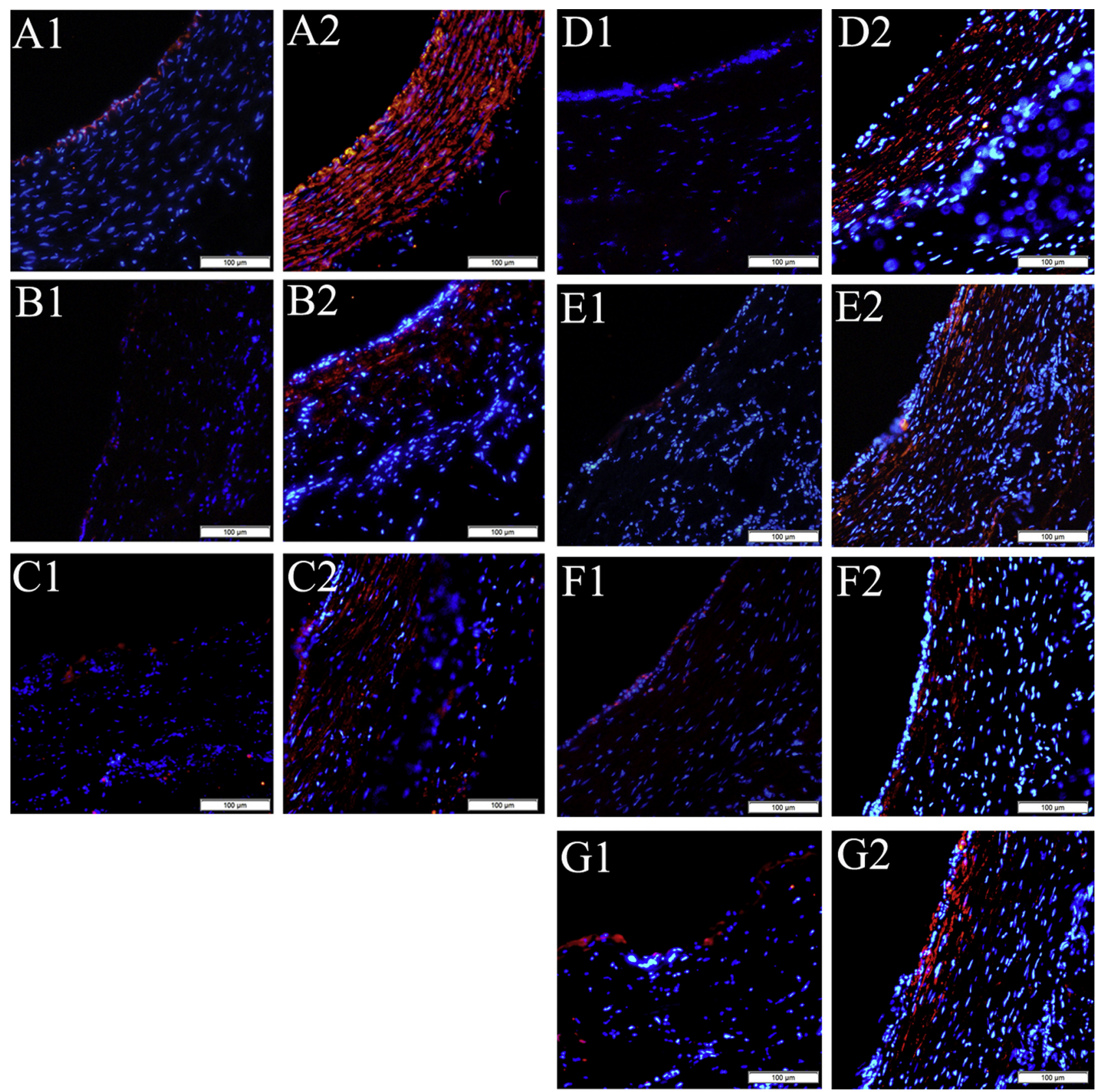

FIGURE 6. CD 31 and $\alpha$-SMA expression in the grafts. Cross-section CD31 immunofluorescence stain (red). A1, Native carotid artery (NCA). B1, Monolayer small intestinal submucosa (S) at 2 months. C1, S at 3 months. D1, Bilayer small intestinal submucosa with curdlan (SC) at 2 months. E1, SC at 3 months. F1, Bilayer small intestinal submucosa with curdlan + dipyridamole (SCD) at 2 months. G1, SCD at 3 months. Endothelial cells were scattered in the lumen at $\mathrm{S}$ at 2 months, $\mathrm{S}$ at 3 months, $\mathrm{SC}$ at 2 months and $\mathrm{SC}$ at 3 months. Endothelial cells were almost continuously distributed in the lumen of NCA, SCD at 2 months, and SCD at 3 months. Cross-section $\alpha$-SMA immunofluorescence stain (red). A2, NCA. B2, S at 2 months. C2, S at 3 months. D2, SC at 2 months. E2, SC at 3 months. F2, SCD at 2 months. G2, SCD at 3 months. SMC layer of grafts at 3 months were all thicker than grafts at 2 months. SMC layer of SCD was thinner than SC at 2 moths and 3 months.

IQR, 1.368-1.521) $(P=.001$ and .003 , respectively $)$ (Figure 7, $D$ and $E$, and Table 4).

\section{DISCUSSION}

Great progress has been made in the application of artificial vascular grafts in the replacement of the large arteries $(>6 \mathrm{~mm}){ }^{14}$ But the long-term patency of small-caliber vascular grafts $(<4 \mathrm{~mm})$ is associated with high incidence of graft failure. ${ }^{15}$ A variety of natural and synthetic scaffolds seeded by arterial cells or differentiated stem cells have aroused great interest. However, the process of their manufacturing is complex, intrusive, and 
TABLE 3. Endothelialization rate comparison between grafts

\begin{tabular}{|c|c|c|c|}
\hline \multirow[b]{2}{*}{ Graft } & \multicolumn{2}{|c|}{ Endothelialization rate $(\%)$} & \multirow[b]{2}{*}{$P$ value } \\
\hline & $2 \mathrm{mo}$ & $3 \mathrm{mo}$ & \\
\hline$S$ & $\begin{array}{l}36.3(32.938 .8) \\
(\mathrm{n}=4)^{*}\end{array}$ & $\begin{array}{l}54.3(50.4-57.1) \\
(\mathrm{n}=6) \dagger\end{array}$ & .019 \\
\hline $\mathrm{SC}$ & $\begin{array}{l}35.9(30.2-42.7) \\
(\mathrm{n}=4) \ddagger\end{array}$ & $\begin{array}{l}55.4(53.7-57.5) \\
(\mathrm{n}=5) \S\end{array}$ & .016 \\
\hline SCD & $\begin{array}{l}79.9(73.5-80.9) \\
(\mathrm{n}=8)\end{array}$ & $\begin{array}{l}90.3(84.4-91.9) \\
(\mathrm{n}=7)\end{array}$ & .006 \\
\hline
\end{tabular}

Values are presented as median (interquartile range). Nonparametric test Mann-Whitney $U$ test was used. $S$, Monolayer small intestinal submucosa; $S C$, bilayer small intestinal submucosa with curdlan; $S C D$, bilayer small intestinal submucosa with curdlan + dipyridamole. $* P=.004$ versus $\mathrm{SCD}$ at 2 months. $\dagger P=.004$ versus SCD at 2 months. $\ddagger P=.001$ versus SCD at 3 months. $\S P=.003$ versus SCD at 3 months. time-consuming. Artificial synthetic vascular grafts that can promote autologous regeneration is believed to be more practical than grafts with seeding. ${ }^{16}$

Our approach was to use decellularized SIS as a basis for small-caliber vascular grafts. The mechanical properties and antienzymolytic properties of biological scaffolds would be significantly reduced by decellularization management. ${ }^{17}$ Therefore, it is necessary to cross-link the decellular matrix to compensate for the negative effects of decellularization. Furthermore, when monolayer SIS was transplanted into the arterial system as an artificial vascular graft or patch, part of them formed aneurysm, ${ }^{18}$ just as observed in our study. Thus, a 2-layer SIS graft was developed to modify mechanical properties.

A
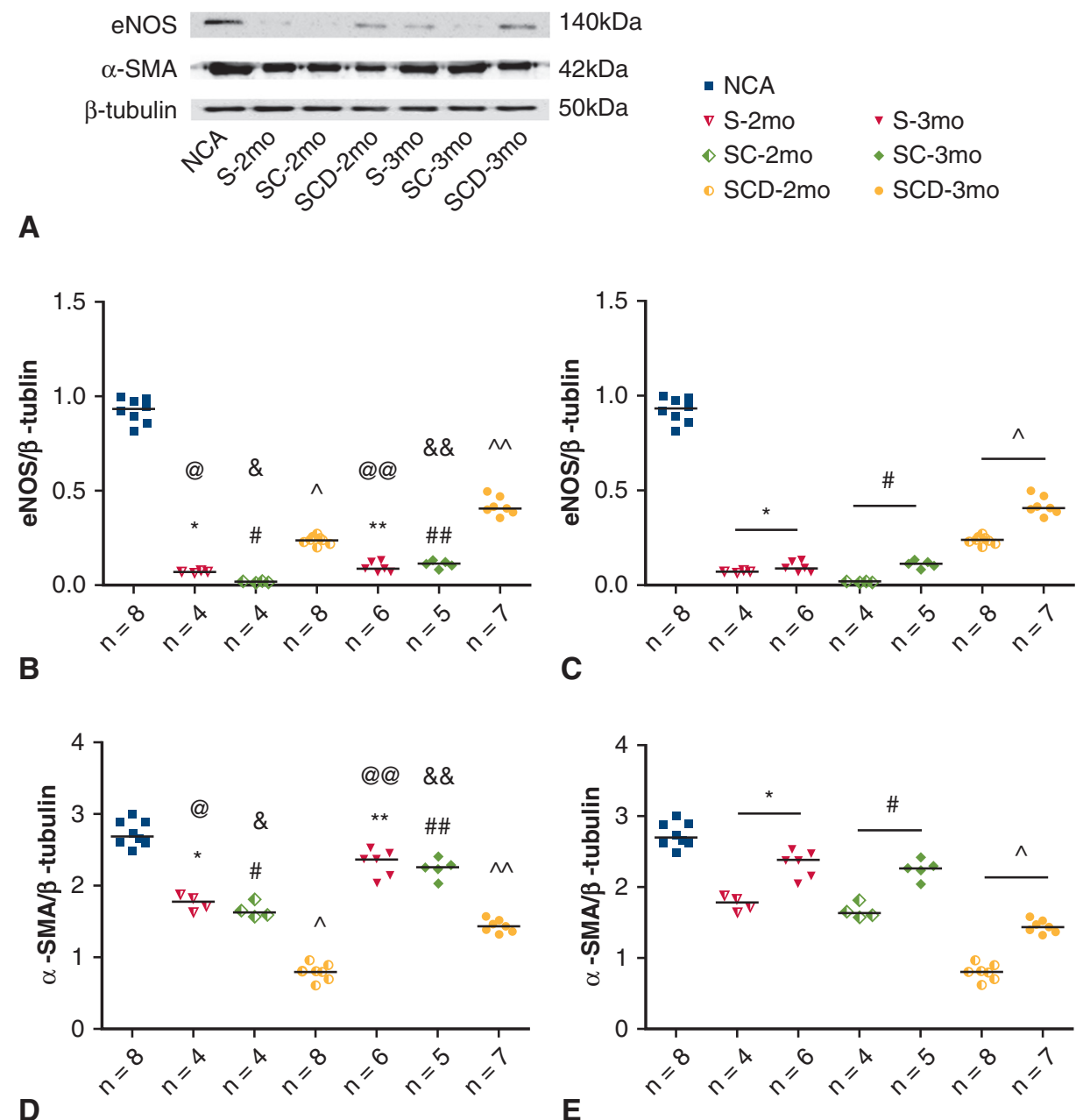

FIGURE 7. A, Western blot analysis of the expression of endothelial nitric oxide synthase (eNOS) and $\alpha$-smooth muscle actin $(\alpha$-SMA) in the grafts. $\beta$-tubulin was used as an internal control. Intensity of eNOS and $\alpha$-SMA was standardized to that of $\beta$-tubulin. Nonparametric Mann-Whitney $U$ test was used for quantitative analysis of the expression of eNOS and $\alpha$-SMA. eNOS expression increased and $\alpha$-SMA decreased in bilayer small intestinal submucosa with curdlan + dipyridamole $(S C D)$ grafts. $\mathrm{B},{ }^{*} P=.004, \# P=.004,{ }^{\wedge} P=.001,{ }^{*} * P=.001, \# \# P=.002$, and ${ }^{\wedge}{ }^{\wedge} P=.001$ versus native carotid artery $(N C A)$ and $@ P=.004, \& P=.004$ versus $\mathrm{SCD}$ at 2 months and $@ @ P=.001, \& \& P=.003$ versus SCD at 3 months. $\mathrm{C}, * P=.171, \# P=.016$, and $\wedge P=.001 . \mathrm{D}, * P=.004, \# P=.004, \wedge P=.001,{ }^{*} * P=.001, \# \# P=.002$, and ${ }^{\wedge} \wedge P=.001$ versus NCA; $@ P=.004, \& P=.004$ versus $\mathrm{SCD}$ at 2 months; and $@ @=.001$ and $\& \& P=.003$ versus $\mathrm{SCD}$ at 3 months. $\mathrm{E},{ }^{*} P=.01, \# P=.016$, and ${ }^{\wedge} P=.001 . S$, Monolayer small intestinal submucosa; $S C$, bilayer small intestinal submucosa with curdlan. 
TABLE 4. Western blot analysis of the expression of endothelial nitric oxide synthase (eNOS) and $\alpha$-smooth muscle actin ( $\alpha$-SMA) in the grafts and native carotid artery (NCA)

\begin{tabular}{lccc}
\hline Variable & Measurement & eNOS/ $\beta$-tubulin & $\alpha$-SMA/ $\beta$-tubulin \\
\hline NCA & 8 & $0.933(0.876-0.981)$ & $2.692(2.608-2.888)$ \\
S 2 mo & 4 & $0.071(0.067-0.075)$ & $1.776(1.679-1.854)$ \\
S 3 mo & 6 & $0.088(0.071-0.122)$ & $2.373(2.145-2.461)$ \\
SC 2 mo & 4 & $0.018(0.015-0.021)$ & $1.627(1.581-1.734)$ \\
SC 3 mo & 5 & $0.112(0.102-0.123)$ & $2.261(2.227-2.294)$ \\
SCD 2 mo & 8 & $0.238(0.225-0.252)$ & $0.803(0.745-0.854)$ \\
SCD 3 mo & 7 & $0.407(0.387-0.471)$ & $1.434(1.368-1.521)$ \\
\hline
\end{tabular}

Values are presented as $\mathrm{n}$ or median (interquartile range). $N C A$, Native carotid artery; $S$, monolayer small intestinal submucosa; $S C$, bilayer small intestinal submucosa with curdlan; $S C D$, bilayer small intestinal submucosa with curdlan + dipyridamole.

Decellularized SIS is an ideal natural scaffold that has low immunogenicity, good mechanical properties, and biocompatibility and eventually can be absorbed or replaced. ${ }^{19}$ In our study, the wall thickness of grafts of SCD-2 months and SCD-3 months was obviously thicker than that of the original. With remodeling, the wall thickness of vascular graft of the SCD-3 months decreased, but the lumen did not change significantly. This was a promising result, as was the development of a neoartery required by the formation of a blood vessel wall. To the contrary, the graft wall of SC-3 months became thicker and the lumen became narrower. Meanwhile the part of S dilated obviously may be due to monolayer SIS structure being insufficient to support mechanical tension in the arterial lumen during hemodynamic and tissue remodeling.

Local delivery of antiproliferative drugs to the injured site to prevent myoproliferative response and avoid systemic medication-related problems has been proven to be effective in reducing stenosis. ${ }^{20,21}$ DIP delivered locally inhibited cell hyperplasia in arteries and intimal thickening after balloon injury. ${ }^{22}$ Sharma and colleagues ${ }^{23}$ found that DIP significantly prevented diabetes mellitus-induced vascular endothelial dysfunction in rats by activating endothelial phosphoinositide 3-kinaseseNOS-nitric oxide signaling pathway. Curdlan is 1 kind of drug delivery carrier; its safety and nontoxicity were evaluated through a series of in vitro and animal experiments. ${ }^{24}$ In our experiment, the graft SCD analysis demonstrated that the release rate of DIP was $53.39 \% \pm 3.52 \%$ at 2 months and $91.01 \% \pm 4.79 \%$ at 3 months. Basically, it has achieved the purpose of drug sustained release.

The early endothelialization in SCD may be due to the specific binding capability of heparin toward Vascular endothelial growth factor and positive effect of DIP to EC proliferation. ${ }^{25}$ We not only identified markers of vascular endothelial differentiation by $\mathrm{CD} 31$, but also evaluated the function of ECs by eNOS, which has the function of vasodilatation and inhibition of platelet aggregation. ${ }^{26}$ Our results showed that the degree of endothelialization of SCD was better than that of SC and S.

Appropriate amount of VSMCs and elastin are markers of muscular artery. Accordingly, VSMCs were aligned regularly in neointima of SCD, compared with disorder arrangement in SC and S. However, no fibrillar elastin was found in any of the grafts, indicating that the remodeling was not complete.

Some limitations exist in our research. The mechanical properties of explantated grafts were not tested. The blood composition of rabbit is not exactly the same of humans, and the internal environments that may cause arteriosclerosis such as hypertension, hyperlipidemia, and hyperglycemia were absent. ${ }^{27}$ The length of the grafts was only $2 \mathrm{~cm}$, which might be different from clinical practice. Further studies examining patency beyond 6 months will be necessary to determine the patency of the grafts when DIP is no longer present.

\section{CONCLUSIONS}

We successfully designed 1 type of hybrid small-caliber artificial vascular graft by making full use of SIS properties of low immunogenicity, degradability, and convenience for surgical manipulation. The 1-ethyl-3(3-dimethylaminopropyl) carbodiimide cross-linking and bilayer SIS structure design modified the mechanical properties of the hybrid graft. Antiplatelet adhesion performance increased by coating with heparin and the drug delivery system of curdlan loaded with DIP acting as the circumferential sandwich filler. Due to all of these, the patency rate of the novel, small-caliber artificial vascular graft in vivo implantation was improved.

\section{Conflict of Interest Statement}

Authors have nothing to disclose with regard to commercial support.

Dr Fang thanks Dr Qiang Ao, Hao Tong and Weijian Hou for providing technical assistance and friendship.

\section{References}

1. Cicha I, Singh R, Garlichs CD, Alexiou C. Nano-biomaterials for cardiovascular applications: clinical perspective. J Control Release. 2016;229:23-36.

2. Sánchez-Palencia DM, Navarro J, Araque JC, Umaña JB, Guerrero AF, Quijano LM, et al. Effects of fabrication on early patency and regeneration of small intestinal submucosa vascular grafts. ASAIO J. 2015;61:596-604.

3. Spicer EJ, Goldenthal EI, Ikeda T. A toxicological assessment of curdlan. Food Chem Toxicol. 1999;37:455-79.

4. Zhang H, Nishinari K, Williams MA, Foster TJ, Norton IT. A molecular description of the gelation mechanism of curdlan. Int J Biol Macromol. 2002; 30:7-16.

5. Chen J, Seviour R. Medicinal importance of fungal beta-(1->3), (1->6)-glucans. Mycol Res. 2007;111:635-52.

6. Wakabayashi Y, Fujita H, Fujimoto K, Kawaguchi H, Morita I, Murota S. Dipyridamole, an anti-platelet agent, reduces the vascular endothelial cell injury induced by active oxygen species. Platelets. 1995;6:176-82. 
7. Punnakitikashem P, Truong D, Menon JU, Nguyen KT, Hong Y. Electrospun biodegradable elastic polyurethane scaffolds with dipyridamole release for small diameter vascular grafts. Acta Biomater. 2014;10:4618-28.

8. Basmadjian D, Sefton MV. Relationship between release rate and surface concentration for heparinized materials. J Biomed Mater Res. 1983;17: 509-18.

9. Abraham GA, Murray J, Billiar K, Sullivan SJ. Evaluation of the porcine intestinal collagen layer as a biomaterial. J Biomed Mater Res. 2000; 51:442-52.

10. Ding Y, Yang M, Yang Z, Luo R, Lu X, Huang N, et al. Cooperative control of blood compatibility and re-endothelialization by immobilized heparin and substrate topography. Acta Biomater. 2015;15:150-63.

11. Iuliano L, Piccheri C, Coppola I, Praticò D, Micheletta F, Violi F. Fluorescence quenching of dipyridamole associated to peroxyl radical scavenging: a versatile probe to measure the chain breaking antioxidant activity of biomolecules. Biochim Biophys Acta. 2000;1474:177-82.

12. Cai N, Koizumi JH, Vazquez MF. Use of 4\% paraformaldehyde in modified Diff-Quik stain and in immunocytochemistry fixation techniques for fine needle aspiration biopsies. Acta Cytol. 2009;53:366.

13. Yavuz K, Geyik S, Pavcnik D, Uchida BT, Corless CL, Hartley DE, et al. Comparison of the endothelialization of small intestinal submucosa, Dacron, and expanded polytetrafluoroethylene suspended in the thoracoabdominal aorta in sheep. J Vasc Intervent Radiol. 2006;17:873-82.

14. Koobatian MT, Row S, Smith RJ Jr, Koenigsknecht C, Andreadis ST, Swartz DD. Successful endothelialization and remodeling of a cell-free small-diameter arterial graft in a large animal model. Biomaterials. 2016;76:344-58.

15. Nagaoka Y, Yamada H, Kimura T, Kishida A, Fujisato T, Takakuda K. Reconstruction of small diameter arteries using decellularized vascular scaffolds. J Med Dent Sci. 2014;61:33-40.

16. Yokota T, Ichikawa H, Matsumiya G, Kuratani T, Sakaguchi T, Iwai S, et al. In situ tissue regeneration using a novel tissue-engineered, small-caliber vascular graft without cell seeding. J Thorac Cardiovasc Surg. 2008;136:900-7.

17. McCready RA, Hodde J, Irwin RJ, Coffey AC, Divelbiss JL, Bryant MA, et al. Pseudoaneurysm formation in a subset of patients with small intestinal submucosa biologic patches after carotid endarterectomy. J Vasc Surg. 2005; 41:782-8.
18. Dobrilovic N, Soukas P, Sadiq I, Goldstein L, Raman J. Early complications of biologic extracellular matrix patch after use for femoral artery repair. $J$ Vasc Surg. 2017;65:705-10.

19. Gilbert TW, Stewart-Akers AM, Simmons-Byrd A, Badylak SF. Degradation and remodeling of small intestinal submucosa in canine Achilles tendon repair. $J$ Bone Joint Surg Am. 2007;89:621-30.

20. Luong-Van E, Grøndahl L, Chua KN, Leong KW, Nurcombe V, Cool SM. Controlled release of heparin from poly(e-caprolactone) electrospun fibers. Biomaterials. 2006;27:2042-50.

21. Edelman ER, Nathan A, Katada M, Gates J, Karnovsky MJ. Perivascular graf heparin delivery using biodegradable polymer wraps. Biomaterials. 2000;21: 2279-86.

22. Singh JP, Rothfuss KJ, Wiernicki TR, Lacefield WB, Kurtz WL, Brown RF, et al Dipyridamole directly inhibits vascular smooth muscle cell proliferation in vitro and in vivo: implications in the treatment of restenosis after angioplasty. J Am Coll Cardiol. 1994;23:665-71.

23. Sharma AK, Khanna D, Balakumar P. Low-dose dipyridamole treatment partially prevents diabetes mellitus-induced vascular endothelial and renal abnormalities in rats. Int $J$ Cardiol. 2014;172:530-2.

24. El-Naggar ME, Abdelgawad AM, Salas C, Rojas OJ. Curdlan in fibers as carriers of tetracycline hydrochloride: controlled release and antibacterial activity. Carbohydr Polym. 2016;154:194-203.

25. McLuckie M, Schmidt CA, Oosthuysen A, Sanchez-Macedo N, Merker H, Bezuidenhout D, et al. High heparin content surface-modified polyurethane discs promote rapid and stable angiogenesis in full thickness skin defects through VEGF immobilization. J Biomed Mater Res A. 2017;105:2543-50.

26. Kader KN, Akella R, Ziats NP, Lakey LA, Harasaki H, Ranieri JP, et al eNOS-overexpressing endothelial cells inhibit platelet aggregation and smooth muscle cell proliferation in vitro. Tissue Eng. 2000;6:241-51.

27. Emini Veseli B, Perrotta P, De Meyer GRA, Roth L, Van der Donckt C, Martinet W, et al. Animal models of atherosclerosis. Eur J Pharmacol. 2017;816:3-13.

Key Words: small intestinal submucosa, curdlan, dipyridamole, small caliber artificial vascular graft 